\title{
Uncertainty over First Stars
}

\author{
New analysis of nuclear reaction data finds holes in a theory about the \\ first stars. \\ By Michael Schirber
}

S tars are dutiful recyclers. The material used and discarded by one generation of stars is taken up and reused by the next. But that cycling process has trouble explaining the surprisingly high calcium concentrations in stars that formed early on. Astrophysicists have proposed that these old stars were seeded by a first generation that expired in "faint supernovae," producing calcium but few other heavy elements. However, a new analysis of nuclear reaction measurements suggests that this model may not be so solid [1].

The first massive stars formed shortly after the big bang and burned brightly but briefly. Astronomers can infer information about this generation by looking at existing stars that were born from their leftovers. These old stars lack heavy elements, suggesting the first stars ended in whimpers rather than bangs. But for this faint-supernova model to explain the calcium data, the first stars would need to have burned hydrogen into calcium through a series of so-called breakout reactions.

James deBoer, from the University of Notre Dame in Indiana, and his colleagues have compiled 70 years of data on a particular breakout reaction in which a fluorine nucleus

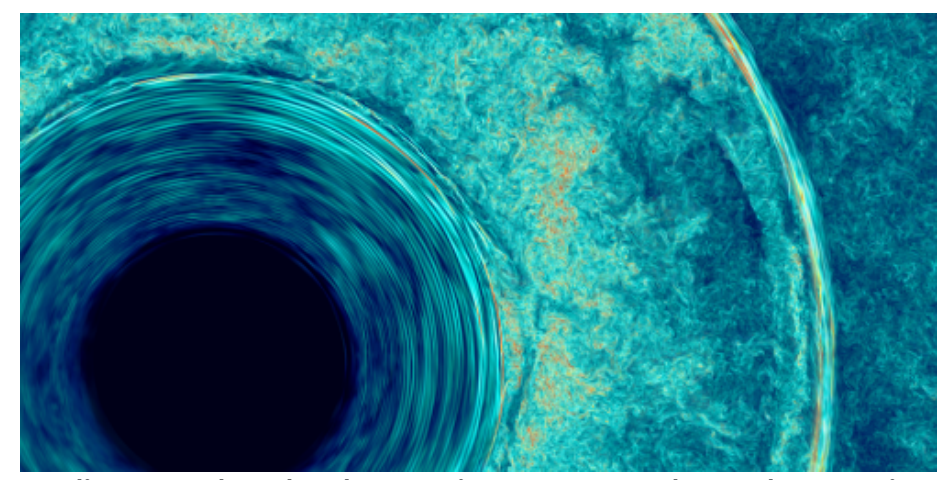

Credit: P. Woodward and F. Herwig; NSF; Texas Advanced Computing Center captures a proton, creating a neon nucleus and a gamma ray. They performed a reaction network analysis, which considered competing reactions. For example, a fluorine nucleus can capture a proton but produce an oxygen nucleus and an alpha particle-a step backward on the path toward calcium. The analysis showed a much larger uncertainty in the fluorine breakout reaction rate than previously reported, casting doubt on the faint-supernova model. The authors say that measurements at lower energy are needed to reduce uncertainty in the reaction rates.

Michael Schirber is a Corresponding Editor for Physics based in Lyon, France.

\section{REFERENCES}

1. R. deBoer et al. " ${ }^{119} \mathrm{~F}(p, \gamma){ }^{20} \mathrm{Ne}$ and ${ }^{19} \mathrm{~F}(p, \alpha \gamma){ }^{16} \mathrm{O}$ reaction rates and their effect on calcium production in Population III stars from hot CNO breakout," Phys. Rev. C 103, 055815 (2021). 